\title{
Interfacial elastic relaxation during the ejection of bi-layered tablets
}

\begin{abstract}
The predilection of a bi-layered tablet to fail in the interface region after its initial formation in the compaction process reduces its practicality as a choice for controlled release solid drug delivery system. Hence, a fundamental appreciation of the governing mechanism that causes the weakening of the interfacial bonds within the bi-layered tablet is crucial in order to improve the overall bi-layered tablet mechanical integrity. This work has shown that the occurrence of the elastic relaxation in the interface region during the ejection stage of the compaction process decreases with the increase in the bi-layered tablet interface strength. This is believed to be due to the increase in the plastic bonding in the interface region. The tablet diametrical elastic relaxation affects the tablet height elastic relaxation, where the impediment of the tablet height expansion is observed when the interface region experiences a diametrical expansion.
\end{abstract}

